\title{
A NONSTANDARD IDEAL OF A RADICAL BANACH ALGEBRA OF POWER SERIES
}

\author{
BY MARC P. THOMAS
}

1. Introduction. We will be concerned here with a result about certain radical Banach algebras of power series. Let $\mathbf{C}[[z]]$ denote the algebra of formal power series over the complex field $\mathbf{C}$. We say that a sequence of positive reals $\{w(n)\}$ is an algebra weight provided the following hold:

$$
\begin{gathered}
w(0)=1 \quad \text { and } 0<w(n) \leq 1 \quad\left(n \in \mathbf{Z}^{+}\right), \\
w(m+n) \leq w(m) w(n) \quad\left(m, n \in \mathbf{Z}^{+}\right) \\
\lim _{n \rightarrow \infty} w(n)^{1 / n}=0 .
\end{gathered}
$$

If these conditions hold it is routine to check that

$$
l^{1}(w(n)) \equiv\left\{y=\sum_{n=0}^{\infty} y(n) z^{n}: \sum_{n=0}^{\infty}|y(n)| w(n)<\infty\right\}
$$

is both a subalgebra of $\mathbf{C}[[z]]$ and a radical Banach algebra with identity adjoined. The norm and multiplication are defined in the natural way (see [3, 4, and 8]). There are obvious closed ideals in $l^{1}(w(n))$ :

$$
M(n) \equiv\left\{\sum_{k=0}^{\infty} y(k) z^{k} \in l^{1}(w(n)): y(0)=y(1)=\cdots=y(n-1)=0\right\}
$$

and, of course, the zero ideal. Such ideals are referred to as standard ideals. Any other closed ideals are denoted nonstandard ideals. It has been an open question for some time whether there exists any algebra weight $\{w(n)\}$ such that $l^{1}(w(n))$ contains a nonstandard ideal [6, p. 189], and the problem seems to go back to Silov (a proposed solution appearing in the literature $[6, \mathrm{p}$. $205]$ is in error). Interest has also been focused upon the quotient algebras $\left(l^{1}(w(n)) / I\right)$, where $I$ is assumed to be nonstandard, since these algebras are representative of all radical Banach algebras with power series generators [1]. Our result is the following:

THEOREM. There exists an algebra weight $\{w(n)\}$ for which the algebra $l^{1}(w(n))$ contains a nonstandard ideal.

Received by the editors February 14, 1983.

1980 Mathematics Subject Classification. Primary 46J20; Secondary 47A15.

(c) 1983 American Mathematical Society $0273-0979 / 83 \$ 1.00+\$ .25$ per page 
In the next section we will sketch a procedure for constructing such an algebra weight $\{w(n)\}$. We will define

$$
x=\sum_{n=1}^{\infty} x(n) z^{n} \in l^{1}(w(n)), \quad x(1) \neq 0,
$$

such that the closed ideal generated by $x$ is nonstandard. If $T$ denotes the operator of right translation on $l^{1}(w(n))$, to prove that this ideal is nonstandard is equivalent to showing that $z \notin \overline{\operatorname{span}}\left\{T^{k} x\right\}_{k=0}^{\infty}$. We note in passing that by weighting the operator rather than the space, one can rephrase the problem in terms of weighted shift operators which are quasinilpotent and strictly cyclic (see [7]).

2. Semimultiplicative weights. Our weight will belong to a special class of weights which are called semimultiplicative. Let $\{n(k)\}$ be a strictly increasing sequence of positive integers with $n(1)=1$. Let the values $w(n(k))$ be assigned subject to the conditions:

$$
w(n(k+1))^{1 /(n(k+1)+n(k))} \leq w(n(k))^{1 / n(k)} \quad(k \in \mathbf{N})
$$

and

$$
\lim _{k \rightarrow \infty} w(n(k))^{1 / n(k)}=0 .
$$

The weight is defined for other values by setting $w(0)=1$ and setting

$$
w(\operatorname{tn}(k)+j)=w(n(k))^{t} w(j) \text { if } \operatorname{tn}(k)+j<n(k+1) .
$$

One says that $\{w(n)\}$ is the semimultiplicative weight generated by $\{w(n(k))\}$. It can be shown that such a weight is an algebra weight [10, Lemmas 2.4 and 2.5]. We will need to specify further conditions on the rate of increase of the sequence $\{n(k)\}$ and require the sequence $\{w(n(k))\}$ to approach zero sufficiently rapidly. When $\{w(n)\}$ has been specified, the element $x$ is chosen to be the lacunary series $x=\sum_{k=1}^{\infty} x(n(k)) z^{n(k)}$, where $x(n(1))=1$ and $|x(n(k))| w(n(k))=2^{-k+1}$ for $k \in \mathbf{Z}^{+}, k \geq 2$. We remark in passing that weights similar to semimultiplicative weights have been shown to possess pathological multiplier algebras [2].

3. Remarks on the proof. Let $A=l^{1}(w(n))$. To show that the closed principal ideal $A x^{-}$is nonstandard, it suffices to prove that there exists $\sigma>0$ such that

$$
\left\|z-\sum_{n=0}^{N} a(n) T^{n} x\right\| \geq \sigma
$$

for all choices of finite sequences $\{a(n)\}_{n=0}^{N}$. Let $\{c(n)\}$ be the associated sequence [9, Definition 2.1], which is the sequence of coefficients for which one has

$$
x * \sum_{n=0}^{N} c(n) z^{n}=z
$$

in the algebra $\mathbf{C}[[z]]$ of formal power series. The series $\sum_{n=0}^{\infty} c(n) z^{n}$ will not, in general, lie in $A$, since many of its coefficients are too large. However, sums 
of the form

$$
x * \sum_{n=0}^{N} c(n) z^{n}=\sum_{n=0}^{N} c(n) T^{n} x
$$

would be natural choices for approximating $z$. It has been shown that, for the type of weights we are considering, an estimate of the form (3.1) does hold for these sums [5]. The key to the proof that (3.1) holds for all finite sums lies in the fact that, if there is a sequence $\left\{\sum_{n=0}^{N_{p}} a^{(p)}(n) T^{n} x\right\}$ which converges to $z$, then for each $k$, the sequence $\left\{a^{(p)}(k)\right\}$ converges to $c(k)$. A contradiction is then reached through a complicated recursive argument, based upon the lacunarity of the element $x=\sum_{k=1}^{\infty} x(n(k)) z^{n(k)}$ and the rate of decrease of the terms $w(n(k))$, which shows that any element $\sum_{n=0}^{\infty} a(n) T^{n} x$ of $A$ which is sufficiently close in norm to $z$ must necessarily have infinitely many nonzero terms.

\section{REFERENCES}

1. G. R. Allan, Commutative Banach algebras with power series generators, Radical Banach Algebras and Automatic Continuity (Proc. Long Beach, 1981), edited by J. M. Bachar et al, vol. 975, Springer-Verlag, 1983.

2. W. G. Bade, H. G. Dales and K. B. Laursen, Multipliers of radical Banach algebras of power series, Mem. Amer. Math. Soc. (to appear).

3. S. Grabiner, Weighted shifts and Banach algebras of power series, Amer. J. Math. 97 (1975), 16-42.

4. K. B. Laursen, Ideal structure in radical sequence algebras, Radical Banach Algebras and Automatic Continuity (Proc. Long Beach, 1981), edited by J. M. Bachar et al, vol. 975, Springer-Verlag, 1983.

5. J. P. McClure, Nonstandard ideals and approximations in primary weighted $l^{1}$-algebras, preprint.

6. N. K. NikolskiY, Selected problems of weighted approximation and spectral analysis, Trudy Mat. Inst. Steklov 120 (1974). Izdat. "Nauka" Leningrad Otdel, Leningrad, 1974; English transl., Proc. Steklov Inst. Math., No. 120 (1974), Amer. Math. Soc., Providence, R.I., 1976.

7. A. L. Shields, Weighted shift operators and analytic function theory, Topics in Operator Theory (C. Pearcy, ed.), Math. Surveys, No. 13, Amer. Math. Soc., Providence, R.I., 1974.

8. M. P. Thomas, Closed ideals and bi-orthogonal systems in radical Banach algebras of power series, Proc. Edinburgh Math. Soc. 25 (1982), 245-257.

9. __ Approximation in the radical algebra $l^{1}\left(w_{n}\right)$ when $\left\{w_{n}\right\}$ is star-shaped, Radical Banach Algebras and Automatic Continuity (Proc. Long Beach, 1981), edited by J. M. Bachar et al, vol. 975, Springer-Verlag, 1983.

10. __ A non-standard closed subalgebra of a radical Banach algebra of power series, J. London Math. Soc. (to appear).

Department of Mathematics, California State College, Bakersfield, CaliFORNIA 93309 
